\title{
Contact precautions for Clostridium difficile and Methicillin-resistant Staphylococcus aureus (MRSA)
}

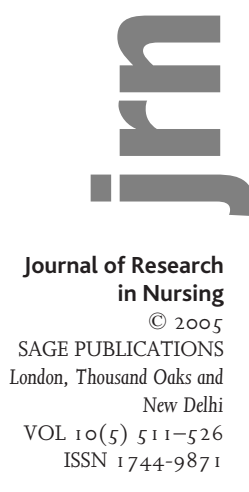

Jacqui Prieto PhD, BSc (Hons), BSc, RGN

Lecturer

School of Nursing and Midwifery, University of Southampton

Jill Macleod Clark, DBE, PhD, BSc, RGN, FRCN

Head of School and Deputy Dean of the Faculty of Medicine, Health and Biological Sciences

University of Southampton

\begin{abstract}
This paper presents an overview of the key findings, key issues and implications emerging from a single case study designed to explore a group of nurses' and healthcare assistants' infection control practice, and to introduce interventions aimed at implementing best practice. The study was undertaken on one hospital ward and the sample comprised all permanently employed nurses and healthcare assistants ( $n=18$ ). Guidelines on Contract Precautions were developed and informed by an expert panel of infection control nurses ( $n=1 \circ 0)$ from across the UK. The detailed investigation of individual participant's responses to the intervention during its implementation provided unique insights into the factors which influence nurses' and healthcare assistants' decisionmaking in relation to infection control practice. Analyses of the data from all three phases of the study revealed that participants experienced great difficulty comprehending infection control recommendations and varied in the extent to which they adopted them. Their capacity to understand and implement these recommendations was hampered, not only by a lack of knowledge, but also by irrational beliefs, inaccurate perceptions of risk, both in relation to themselves and patients, and a lack of ability or willingness to exercise clinical judgement,
\end{abstract}


Journal of Research in Nursing I o(5)

particularly in relation to glove use. These findings highlight the need for further study in the drive to improve this crucial aspect of health care services.

Key words Clostridium difficile, contact precautions, infection control practice, MRSA, nurses' perceptions, supportive intervention, professional development

\section{Background}

Healthcare-associated infection (HAI) is a complication which affects approximately $9 \%$ of patients admitted to hospitals in the United Kingdom (UK) (National Audit Office, 2000). This is estimated to cost the UK National Health Service (NHS) £ I billion per year (National Audit Office, 2000). The threat to public health posed by antibiotic-resistant bacteria such as Methicillin-resistant Staphylococcus aureus (MRSA) and other pathogens such as Clostridium difficile is an ongoing concern (Department of Health, 2003; National Audit Office, 2004). Recently the UK Government has focused increased attention to the problem of HAI, identifying the need for intensified control measures (Department of Health, 2003, 2004).

The routine use of infection control procedures by healthcare practitioners in clinical practice is crucial to the prevention of healthcare-associated infection (Pratt et al., 200I). However, within healthcare there is a welldocumented history of poor adherence to infection control practices such as hand hygiene (Pittet, 2000) and the use of personal protective equipment (Levin, I 995). Moreover, intervention studies designed to address the problem have largely met with limited success (Pittet, 2000). To date, there has been limited exploration of healthcare practitioners' own perspectives of this issue and their perceptions of the priorities for practice development have rarely formed the basis of intervention studies designed to improve practice. Typically, intervention studies have focused upon outcome measures, yet have been unable to explain their findings in any detail, owing to a lack of exploration of the process of changing practice.

\section{Introduction}

This paper describes a detailed investigation of the infection control practices of nurses and healthcare assistants on one hospital ward. The aims of the study were:

- To identify study participants' priorities, views and anxieties about infection-control practice and observe the nature and extent of the problems identified

- To design and implement a supportive intervention to improve practice and monitor individual participants' responses to the change process 
Prieto and Clark Clostridium difficile and Methicillin-resistant Staphylococcus aureus

- To determine participants' self-reported changes in practice and their perceptions of the impact of the supportive intervention

\section{Method}

The study comprised a detailed investigation of the infection control practices of nurses and healthcare assistants on one general medical ward. It involved the design and implementation of a supportive intervention to improve practice and was divided into three phases: pre-implementation (Phase I), implementation (Phase II) and post-implementation (Phase III). An outline of the study design is shown in Figure I. In Phase I, nurses and healthcare assistants were interviewed using a semi-structured interview schedule to explore their views and anxieties about infection control practice and identify their priorities for practice development. The findings of Phase I informed the design of an education and support programme to improve practice, involving practice guidelines on Contact Precautions and the provision of one-to-one instruction and support during their implementation. In Phase II of the study, participant observation of practice was undertaken in order to assess the impact of the supportive intervention. Semi-structured interviews with the nurses and healthcare assistants were carried out in Phase III to gain insights into the participants' perceptions of the intervention and its effect on their practice. Six of the i 9 nurses and healthcare assistants included in Phase I were no longer employed on the ward by Phase II. However, all i 8 of those involved in Phase II were followed up in Phase III. Ethical approval to undertake the study was obtained from the local ethics committee and informed consent gained from all those engaged in the study.

\section{The study ward}

The study ward was located within a district general hospital, which was one of four hospitals comprising an acute National Health Service Trust. It was a 22-bed male medical ward catering predominantly for healthcare of the elderly patients in need of non-acute medical intervention. The only prerequisites identified for its suitability were an interest amongst the ward manager and staff in developing infection control practice and willingness to actively engage in the research. There were no particular requirements with regard to infection control, since this is a fundamental aspect of clinical practice on all hospital wards. Selection of the ward was guided initially by nursing management and subsequently by consultation with the ward manager and her nursing team, all of whom expressed their support. 
Journal of Research in Nursing I O (5)

\section{Figure 1 Outline of Study Design}

\section{Phase I: Pre-implementation}

Stage 1 Semi-structured interviews

Semi-structured interviews with all nurses and healthcare assistants (19) including ranking exercise

Stage 2 Development of structured observation tool

Development and piloting of structured observation tool to monitor nurses' and healthcare assistants' infection control practice in relation to patients nursed in isolation due to CDAD and MRSA

Structured observations of practice

60 hours of structured observations of nurses' and healthcare assistants' infection control practice in relation to Contact Precautions

Stage 3 Development of practice guideline

Development of practice guidelines on Contact Precautions for patients with CDAD and MRSA, including peer review of content

\section{Phase II: Implementation}

Stage 4 Implementation of practice guideline Implementation of the practice guidelines during a six-month period with oneto-one support and instruction given to all nurses and healthcare assistants (18)

Participant observations of practice Participant observations of individual nurses' and healthcare assistants' infection control practice in relation to Contact Precautions, and their reflections upon this

Record of all questions, comments and concerns raised by study participants in relation to the practice guidelines during their implementation

\section{Phase III: Post-implementation}

Stage 5 Semi-structured interviews

Semi-structured interviews with all nurses and healthcare assistants (18) who participated in Phase II

\section{Data Collection: Phase I}

\section{Semi-structured interviews}

An interview schedule structured around io aspects of infection control practice, which commonly form the basis of hospital infection control policies (Philpott-Howard and Casewell, I 994; Wilson, I 995), was developed and piloted prior to use in the main study. Between January 
Prieto and Clark Clostridium difficile and Methicillin-resistant Staphylococcus aureus and March I997, tape-recorded interviews were conducted with all nurses and healthcare assistants permanently employed on the study ward $(n=$ I 9$)$ to explore their views and anxieties about infection control practice and identify key areas for practice development. Participants were asked to rank the following practices according to the extent to which they perceived there to be difficulty with achieving a good standard of practice:

- aseptic technique

- clinical waste and sharps handling and disposal

- decontamination of equipment

- hand hygiene

- intravascular therapy care

- isolation precautions

- linen handling and disposal

- universal precautions (to protect staff)

- urinary catheter care

- use of gloves and aprons (to protect patients)

The reasons for these difficulties were then explored using an 'open' style of questioning. The findings of the rank-order exercise were analysed quantitatively to produce a mean average ranking of each infection control practice. The interview transcripts were analysed qualitatively to identify the obvious and recurrent issues emerging from the data.

Structured observation of practice The structured observation of practice focused on the observation and description of nurses' and healthcare assistants' infection-control practice in relation to Contact Precautions for patients nursed in single-room isolation due to MRSA and Clostridium difficile associated diarrhoea (CDAD), since this was the primary concern identified by respondents during the interviews. An observation schedule to record sequentially the patient-care activities undertaken and Contact Precautions used during nurse-patient interactions was developed and piloted prior to use in the main study. Between October I 997 and July I 998,60 hours of observation were conducted on 30 separate days. This took place between the hours of $08.00 \mathrm{am}$ and $\mathrm{I} 0.00 \mathrm{am}$, since it had been established during pilot work that this was the most productive time to observe a wide range of patient-care activities representative of those typically undertaken by nurses and healthcare assistants. Independent observations of $20 \%$ of the sample were undertaken to establish the interobserver reliability of the tool.

The process of analysing the data involved making an assessment of the appropriateness of observed practice in relation to the local infection control policy and research-based evidence. For example, hand hygiene 
Journal of Research in Nursing I o(5)

was expected immediately prior to invasive procedures, after contact with the patient or their environment, after visible soiling or contact with a source of micro-organisms and after removing gloves.

Qualitative and quantitative analysis of these data revealed that respondents' concerns related primarily to the use of Contact Precautions for patients with MRSA CDAD. Structured observations of Contact Precautions practice provided an understanding of the prevalence and context of these issues and identified further issues.

Development of practice guidelines on contact precautions In view of the fact that participants considered the existing infection control policy to be lacking in detail, practice guidelines on Contact Precautions were produced to support the intervention. They were written in consultation with study participants and the local infection control team and were informed by the latest research-based evidence. The final draft was subjected to a process of peer review, by an expert panel of infection control nurses ( $\mathrm{n}=\mathrm{I} \circ \mathrm{O})$, from across the United Kingdom. There was complete consensus from the expert panel on all elements of the guidelines with the exception of the useage of gloves and aprons. Here, there was a lack of agreement about whether gloves and aprons should be worn routinely for all patient contact or on a task-specific basis.

\section{Data collection: Phase II}

Participant observation of practice Data collection during Phase II of the study comprised participant observation of practice relating to the care of patients nursed in contact isolation due to CDAD and MRSA. Between January and June I 999, the researcher worked alongside individual study participants $(\mathrm{n}=\mathrm{I} 8)$ during 30 nursing shifts (early, late and night duties) to offer support, practical instruction and performance feedback on implementing the new Contact Precautions guideline. An observation schedule based on nine key areas of the guideline (see Table 2) was used to record individual participants' practice as the researcher worked with them. All questions, comments and concerns that were raised during the course of each shift were recorded in a process record. At the end of each shift, the researcher engaged individual participants in a reflective conversation to provide feedback and encourage reflection on their practice. Participants' responses to this were recorded in the process record. The observations of practice were analysed to determine the extent to which participants' practice was in accordance with the new practice guideline. The process record data were analysed to identify key issues influencing nurses' and healthcare assistants' use of Contact Precautions. 
Prieto and Clark Clostridium difficile and Methicillin-resistant Staphylococcus aureus

Table 1 Interview data: themes arising from the analysis of interview data on Contact Precautions

\begin{tabular}{|c|c|c|}
\hline $\begin{array}{l}\text { 1. Confusion and conflict } \\
\text { about Contact Precautions }\end{array}$ & $\begin{array}{l}\text { 2. Other issues/concerns } \\
\text { about Contact Precautions }\end{array}$ & $\begin{array}{l}\text { 3. Measures required to } \\
\text { promote good practice }\end{array}$ \\
\hline $\begin{array}{l}\text { - Inadequate knowledge base } \\
\text { - Lack of information } \\
\text { - Uncertainty about rationales } \\
\text { for practice } \\
\text { - Inconsistent practice } \\
\text { - Doubts about the } \\
\text { effectiveness of Contact } \\
\text { Precautions } \\
\text { - Risks to staff health }\end{array}$ & $\begin{array}{l}\text { - Non-adherence to } \\
\text { Contact Precautions } \\
\text { - Inadequate isolation } \\
\text { facilities } \\
\text { - Detrimental effects } \\
\text { on patients }\end{array}$ & $\begin{array}{l}\text { - Educational input } \\
\text { - Practice guideline } \\
\text { - Support with practical } \\
\text { implementation }\end{array}$ \\
\hline
\end{tabular}

\section{Data collection: Phase III}

Semi-structured interviews An interview schedule was developed which employed an 'open' style of questioning to elicit individuals' selfreported changes in practice, and perceptions of the impact of the supportive intervention on this. Between January and March 2000, six months after completion of Phase II of the study, tape-recorded interviews were conducted with all participating nurses and healthcare assistants $(n=18)$. Four participants who had left the ward before six months were interviewed on leaving their posts. The interview transcripts were analysed qualitatively to identify the obvious and recurrent issues emerging from the data.

\section{Key Findings}

\section{Phase I: Interview data}

Analysis of the data from the interviews conducted in Phase I revealed that nurses' and healthcare assistants' primary concerns about infection control centred on the isolation precautions required when nursing patients with CDAD and MRSA, the two infectious conditions most frequently encountered on the study ward. In this paper, the more accurate term 'Contact Precautions' is used, since both CDAD and MRSA fall into this category of isolation precautions (HICPAC, I 996). Findings relating specifically to Contact Precautions are now considered, as the priority given to this aspect of infection control practice far outweighed concerns about all other practices.

Table I presents a summary of the emergent themes identified during the analysis under three main headings. The central issue that emerged from the data was the huge confusion and conflict that was perceived to exist amongst staff on the study ward regarding the correct way to implement Contact Precautions. Misconceptions about how infection is 
Journal of Research in Nursing I o(5)

spread were commonplace among both nurses and healthcare assistants. For example, the majority of respondents thought that infection and, in particular, MRSA, is spread mainly by the airborne route rather than by contact. This resulted in a lack of confidence in measures such as hand hygiene and use of gloves and aprons as the means by which to interrupt the transmission of infection. Coupled with this was considerable anxiety amongst respondents about the risk to their own health from exposure to Clostridium difficile and MRSA.

Uncertainty about the rationales for infection control practice and frustration about the resultant inconsistencies in practice led respondents to identify the need for educational input in the form of practice guidelines, since the existing infection control policy was considered to be lacking in detail. The need for some kind of practical supervision was also identified so that questions could be answered as they arose in practice.

\section{Phase I: Structured observations of practice}

In all, 60 hours of observation were conducted, I o of which involved a second observer to assess the inter-observer reliability of the observation schedule. The local infection control policy was very loosely worded and related to procedures before and after care but not during care. It was followed on three-quarters of occasions both by nurses and healthcare assistants. Other than for minimal interactions with patients, such as passing items into the isolation room, protective clothing was generally donned prior to entering the room and was only removed on leaving the room. Hands were usually washed on leaving the room. Whilst, essentially, this was in accordance with the local infection control policy, there were serious deficiencies in hand-hygiene practice during patient care, both by nurses and healthcare assistants. More specifically, gloves were never changed nor hands washed during care when gloved hands had become soiled following contact with a source of micro-organisms.

This was a result of the non-task-specific approach to glove use, which fulfilled the wearer's need to protect themselves from exposure to the patient's micro-organisms. However, it did not allow for a reduction of risks to the patient by ensuring the removal or changing of gloves and washing of hands between 'dirty' and 'clean' procedures. On the occasions when gloves were changed, this occurred just before leaving the patient's room to take a bedpan to the sluice, thus leaving the room provided the cue. This lack of attention to basic hand hygiene principles clearly needed to be addressed during Phase II of the study.

Observations relating to the disposal of linen and clinical waste by nurses and healthcare assistants revealed that this was always segregated correctly, but was removed from the room in a more elaborate way than necessary (Maki et al., I 986), using a 'double-bag' technique. This 
Prieto and Clark Clostridium difficile and Methicillin-resistant Staphylococcus aureus

involved placing a tied linen/waste bag into a second bag held open by a colleague standing outside the room. The removal of bedpans and urine bottles involved placing these items into a clinical waste bag, rather than using one of the paper covers available for this purpose. On several occasions, these too were double-bagged by a colleague at the door threshold in much the same way as for linen and clinical waste bags. This over-elaborate approach to the disposal of linen and waste reflected concerns raised at interview about the potential for airborne spread of infection.

\section{Phase II: Implementation data}

Thirteen nurses and five healthcare assistants participated in Phases II and III of the case study. In all, I 39 questions were asked, the majority of which were concerned with clarifying recommendations within the guidelines immediately prior to implementing them. Questions were also asked about the risk of infection to staff members when nursing patients with CDAD and MRSA, the mode of transmission of these organisms and the effectiveness of Contact Precautions in preventing the spread of infection.

The guidelines recommended the use of gloves and aprons for specific activities according to the likelihood and extent of microbial contamination, rather than their use indiscriminately for virtually all interactions with patients and their environment. This generated a high proportion of entries in the process record (4 I \%) as participants were keen to clarify the activities that required protective clothing from those that did not. The majority of participants ( $n=$ I 2 ) embraced this more selective approach to glove and apron use, although they found it difficult to determine when protective clothing was needed. They also recognised the importance of removing or changing soiled gloves and washing hands during as well as after patient care. However, the remaining participants $(n=6)$ were less willing to adopt this approach, as they remained anxious about the need to protect themselves from infection and were less keen to consider the rationales for glove and apron use in any detail.

These findings from the process record corresponded with the participant observations of practice, as summarised in Table 2. As the table shows, on the majority of occasions, hands were washed and gloves were changed when indicated during care. Likewise, there was a high level of adherence to procedures concerning the disposal of linen and clinical waste and cleaning of equipment. Changes concerning the selective use of gloves occurred less frequently for the reasons indicated above.

A finding that emerged repeatedly from the analysis of Phase II data was the perceived importance of infection control precautions when nursing isolated patients as compared with other patients. Indeed, nurses and healthcare assistants frequently identified that they considered infection control measures to be of greater importance when nursing 
Journal of Research in Nursing I O (5)

Table 2 Researcher's observations of nurses' and health care assistants' practice in relation to Contact Precautions during Phase II

\begin{tabular}{|c|c|c|c|c|c|c|}
\hline \multirow[b]{2}{*}{$\begin{array}{l}\text { Contact Precautions as per } \\
\text { practice guideline }\end{array}$} & \multicolumn{2}{|l|}{ RNs } & \multicolumn{2}{|c|}{ HCAs } & \multicolumn{2}{|l|}{ Total } \\
\hline & Yes & No & Yes & No & Yes & No \\
\hline $\begin{array}{l}\text { Always washed hands during } \\
\text { patient care when indicated }\end{array}$ & 12 & 5 & 2 & 1 & 14 & 6 \\
\hline $\begin{array}{l}\text { Always washed hands at the } \\
\text { end of patient care }\end{array}$ & 17 & 7 & 4 & 2 & 21 & 9 \\
\hline $\begin{array}{l}\text { Gloves and aprons always } \\
\text { worn when indicated }\end{array}$ & 24 & 0 & 6 & 0 & 30 & 0 \\
\hline $\begin{array}{l}\text { Used gloves and aprons } \\
\text { selectively for patient care } \\
\text { activities }\end{array}$ & 12 & 12 & 2 & 4 & 14 & 16 \\
\hline $\begin{array}{l}\text { Always changed gloves } \\
\text { during care when soiled }\end{array}$ & 14 & 3 & 1 & 2 & 15 & 5 \\
\hline $\begin{array}{l}\text { Used single bag system for } \\
\text { disposal of linen }\end{array}$ & 14 & 0 & 5 & 0 & 19 & 0 \\
\hline $\begin{array}{l}\text { Used single bag system for } \\
\text { disposal of clinical waste }\end{array}$ & 7 & 0 & 4 & 0 & 11 & 0 \\
\hline $\begin{array}{l}\text { Did not wrap bedpan/urine } \\
\text { bottle in clinical waste bag }\end{array}$ & 15 & 1 & 5 & 1 & 20 & 2 \\
\hline $\begin{array}{l}\text { Equipment always cleaned } \\
\text { correctly after use }\end{array}$ & 7 & 2 & 3 & 0 & 10 & 2 \\
\hline Total & 122 & 30 & 31 & 11 & $\begin{array}{l}154 \\
(79 \%)\end{array}$ & $\begin{array}{l}40 \\
(21 \%)\end{array}$ \\
\hline
\end{tabular}

isolated patients than other patients and were more likely to adhere to them as part of Contact Precautions than routinely for all patients.

Interestingly, study participants differed in the extent to which they engaged in Phase II of the study. Two-thirds (12/18) of the individuals involved exhibited what the researcher considered to be a high degree of engagement. This included eight nurses and four healthcare assistants, all of whom took the opportunity to fully exploit the support offered to them. These individuals were willing to openly scrutinise and reflect upon their practice. They asked more questions and were more frequently observed to exhibit good practice with regard to Contact Precautions. Conversely, the other six study participants (five nurses and one healthcare assistant) were considered by the researcher to have engaged to a lesser extent. Although none of these individuals actually declined to work with the researcher, they all seemed uncomfortable about it. They also appeared reticent about discussing their practice and made few sponta- 
Prieto and Clark Clostridium difficile and Methicillin-resistant Staphylococcus aureus

Table 3 Self-reported changes in practice as a result of the supportive intervention

\begin{tabular}{|c|c|c|c|c|c|c|}
\hline & \multicolumn{2}{|c|}{ RNs (13) } & \multicolumn{2}{|c|}{ HCAs (5) } & \multicolumn{2}{|l|}{ Total } \\
\hline & Yes & No & Yes & No & Yes & No \\
\hline Washing hands more & 8 & 5 & 3 & 2 & 11 & 7 \\
\hline $\begin{array}{l}\text { Selective use of gloves and } \\
\text { aprons for physical patient } \\
\text { contact }\end{array}$ & 7 & 6 & 2 & 3 & 9 & 9 \\
\hline $\begin{array}{l}\text { Changing gloves during care } \\
\text { when soiled }\end{array}$ & 8 & 5 & 4 & 1 & 12 & 6 \\
\hline $\begin{array}{l}\text { Instructing visitors about } \\
\text { correct precautions to use }\end{array}$ & 11 & 2 & 4 & 1 & 15 & 3 \\
\hline $\begin{array}{l}\text { Using single bag system for } \\
\text { linen and waste disposal }\end{array}$ & 12 & 1 & 5 & 0 & 17 & 1 \\
\hline $\begin{array}{l}\text { Not wrapping bedpans and } \\
\text { urine bottles in yellow bags }\end{array}$ & 7 & 6 & 4 & 1 & 11 & 7 \\
\hline Cleaning equipment correctly & 10 & 3 & 5 & 0 & 15 & 3 \\
\hline Total & 63 & 28 & 27 & 8 & $\begin{array}{l}90 \\
(71 \%)\end{array}$ & $\begin{array}{l}36 \\
(29 \%)\end{array}$ \\
\hline
\end{tabular}

neous comments, often only commenting in response to feedback from the researcher during the reflective feedback session.

\section{Phase III: Interview data}

Table 3 presents a summary of the interview findings relating to selfreported changes in practice as a result of the supportive intervention. As the table shows, the proportion of practices that were reported to have changed as a result of the supportive intervention was 90/ I 26 (7 I\%). Health care assistants reported a slightly higher proportion of changes overall (27/35) as compared with nurses (63/9i). There were variations according to each aspect of practice. For example, almost all (i 7/ i 8) respondents reported using the single bag system for disposal of linen and waste, whereas only half (9/18) reported using gloves on a selective rather than routine basis.

Qualitative analysis of the interview data revealed some reasons why individual participants did or did not change their practice. Eleven respondents that considered they were washing their hands more frequently than prior to the intervention, since they had a greater appreciation of the importance of hand hygiene, both as a Contact Precaution and as part of routine infection-control practice. As one nurse said:

I think before it was very much you only washed your hands if you thought yuck, I've got something on my hands, I'll go and wash them. I'm sure I did 
Journal of Research in Nursing I $\circ(5)$

wash my hands, but it would only be if they were obviously soiled. So yes, from the point of view of just thinking about transferring germs from one patient to another, I wash my hands after each patient no matter what I've done, whereas I wasn't doing that before.

The remaining seven respondents, who reported no change in behaviour, considered that they had always adopted good hand hygiene practice. Interestingly, six of these did not appear to appreciate the importance of changing soiled gloves and washing hands during as well as after patient care when asked about this during the interview. Those respondents who continued to use gloves and aprons routinely rather than selectively expressed ongoing concerns about the risk to themselves from exposure to CDAD and MRSA.

Several respondents expressed an ongoing inclination to direct their infection control efforts more towards patients nursed in isolation than other patients, as found in Phases I and II of the study. As one nurse said:

People are more conscious when they're in there [isolation room] that they're hands must be washed properly, but I feel when you're on the main ward, obviously you've still got to have clean hands, but I find, I even do it myself sometimes, you're in a rush, somebody else is calling you and hands don't always get washed.

Analysis of the interview data relating to the evaluation of the supportive intervention indicated that one-to-one support and instruction was considered by all but three respondents to have influenced their practice. One of the key benefits of this approach was considered to be its applicability to practice, since being able to ask questions that arose while working allowed participants to learn in a very practical, 'hands-on' way. It also enabled any practical difficulties to do with implementing the guideline to be considered in a situational context, giving added credibility to the advice and information imparted by the researcher.

In contrast to one-to-one support and instruction, only two respondents (one nurse and one healthcare assistant) considered the practice guidelines to have been influential. This was despite it having been tailormade at the request of study participants. The main reason given for lack of use of the guideline and, indeed, guidance documents in general was lack of time, particularly as there were so many policies and guidelines that healthcare staff were expected to implement. The fact that guidelines require interpretation in practice was another reason why written information of this kind was not considered to be useful, it being far easier to ask the advice of an experienced colleague. The summarised version of the guideline that was produced as an $\mathrm{A}_{4}$ poster for display as an 'aide-memoire' was considered easy to refer to and helpful by over half ( I I / i 8) of the respondents. Although research articles that supported 
Prieto and Clark Clostridium difficile and Methicillin-resistant Staphylococcus aureus the rationales for recommendations in the practice guideline were made accessible to study participants throughout the duration of the intervention phase, only two nurses considered this to have helped develop their practice. As with the written guideline, the main reasons for the limited impact of research articles were lack of time and the need to interpret them before they can be applied in practice.

\section{Discussion}

A key finding of this study was the immense difficulty perceived by nurses and healthcare assistants in relation to implementing Contact Precautions for patients with CDAD and MRSA. This far outweighed their concerns about all other aspects of infection-control practice, including hand hygiene, despite the problem of poor-adherence to hand-hygiene practice being well recognised in the literature (Pittet, 2000). This finding can be explained in part by participants' misunderstanding of the need for infection-control practice to be focused primarily on those patients with recognised infectious conditions rather than all patients. This was further compounded by there being huge confusion about the rationales for practice in relation to Contact Precautions as well as heightened anxiety about the risk to healthcare workers from exposure to infection.

In this case study, structured observations were only undertaken of the infection control practices employed when nursing isolated patients. In view of this, it was not possible to confirm participants' self-reported practice in relation to their limited use of infection-control measures for patients other than those nursed in isolation.

The difficulties experienced by participants with regard to their confusion about the rationales for infection-control practice and the resultant inconsistencies and rituals that manifested could be explained at least in part by their lack of knowledge of basic microbiology and infectioncontrol principles. The vague and inaccurate accounts they offered about how micro-organisms spread and the misconceptions they held are indicative of this. Courtenay ( I 998) reported similar findings from interviews undertaken with seventeen nurses and healthcare assistants, the majority of whom had an inaccurate understanding of how infection is spread. This included misconceptions about microbiological principles regarding the motility, spread and survival of micro-organisms, which resulted in ritualistic and inconsistent practice.

In this study, educational support was offered in the form of one-to-one practical instruction in the clinical setting. Individuals were encouraged to scrutinise and reflect on their practice while they were working to facilitate learning in a situational context. It also provided opportunities to identify 
Journal of Research in Nursing I o(5)

practitioners' own beliefs about infection control and to challenge their misconceptions. This approach was positively evaluated by the majority of participants owing to its immediate applicability to practice and resulted in changes in their practice. However, it was labour-intensive and even with this high degree of support, individuals still struggled in varying degrees to make decisions about the infection-control measures required in different situations, since they found it difficult to conceptualise how germs spread. Moreover, they did not always respond rationally to the information presented to them.

Participants' anxiety about the risk to their own health and that of their families from exposure to Clostridium difficile and MRSA was found to exert a major influence on their infection control practice. Indeed, this frequently appeared to be the main motivator to adhere to Contact Precautions. Even though the risks to healthcare workers from Clostridium difficile and MRSA are considered to be negligible (BSAC/HIS/ICNA I 998; National Clostridium difficile Standard Group, 2004), a few participants remained entirely unconvinced about this at the end of the study, as revealed during the post-implementation interviews.

\section{Conclusion}

The challenge of promoting optimal adherence to infection control in healthcare practice is a hugely complex one. The difficulties experienced by participants in the present study shed light on some of the possible reasons for this. Whilst the generalisability of these findings may be limited, the issues raised should not be ignored given the extent to which nurses' and healthcare assistants' capacity to understand and implement infection-control practice was hampered, not only by a lack of knowledge, but also by irrational beliefs, inaccurate perceptions of risk, both in relation to themselves and patients, and a lack of ability or willingness to exercise clinical judgement, particularly in relation to glove use.

There is an urgent need to determine whether the findings of the present study are of relevance more generally to nurses and other healthcare workers. If this is the case, a radical rethink will be needed about the ways in which healthcare workers are educated and supported on infectioncontrol issues in practice. Further research in this area is essential to inform the development of interventions to address the problem of poor adherence to infection control practice. For example, it may be that attempts to improve adherence to practices such as hand hygiene will continue to be limited until healthcare workers become more convinced of their value in routine practice as well as in the context of isolation precautions. 


\section{Key points}

- The nurses and healthcare assistants involved in this study experienced huge confusion and conflict surrounding the use of Contact Precautions for CDAD and MRSA

- Study participants' anxiety about the risk to their own health from Clostridium difficile and MRSA was found to exert a major influence on their infection control practice

- One-to-one practical instruction influenced practice to a much greater extent than tailor-made practice guidelines

- Further research is needed to understand healthcare workers' perspectives of the problems associated with implementing infection control practice

\section{References}

British Society for Antimicrobial Chemotherapy/Hospital Infection Society/Infection Control Nurses' Association (BSAC/HIS/ICNA) ( I 998). Revised guidelines for the control of methicillin-resistant Staphylococcus aureus infection in hospitals working party report. Journal of Hospital Infection 39: 253-290.

Courtenay, M. ( I 998) The teaching, learning and use of infection control knowledge in nursing. NT Research 3:2, I I 8-I 30 .

Department of Health (2003) Winning ways. Working together to reduce healthcare associated infection in England. Report from the Chief Medical Officer. London: Department of Health Publications.

Department of Health (2004) Towards cleaner hospitals and lower rates of infection. A summary of action. London: Department of Health Publications.

Hospital Infection Control Practices Advisory Committee (HICPAC) ( 1 996) Guideline for isolation precautions in hospitals. Part II. Recommendations for isolation precautions in hospitals. American Journal of Infection Control 24: I, 32-52.

Levin, P.F. ( 1 995) Improving compliance with universal precautions. American Association of Occupational Health Nurses Journal 43:7, 362-370.

Maki, D.G., Alvarado, C., Hassemer, C. ( 1986 ) Double-bagging of items from isolation rooms is unnecessary as an infection control measure: a comparative study of surface contamination with single and double-bagging. Infection Control 7: i I, 535-537.

National Audit Office (2000) The management and control of hospital-acquired infection in acute NHS Trusts in England: report by the Comptroller and Auditor General. London: Stationery Office.

National Audit Office (2004) Improving patient care by reducing the risk of hospital-acquired infection: a progress report. London: Stationery Office.

National Clostridium difficile Standards Group (2004) National Clostridium difficile Standards Group: Report to the Department of Health. Journal of Hospital Infection 56(Suppl I ): I -38 .

Philpott-Howard, J., Casewell, M. ( I 994) Hospital Infection Control. Policies and Practical Procedures London: WB Saunders Company Ltd. 
Journal of Research in Nursing $\mathrm{I}$ O(5)

Pittet, D. (2000) Improving compliance with hand hygiene in hospitals. Infection Control and Hospital Epidemiology 21 :6, 38 I -386.

Pratt, R., Pellowe, C., Loveday, HP., Robinson, N., Smith, G.W. and the epic guideline development team (200 I) The epic project: developing national evidence-based guidelines for preventing healthcare associated infections. Journal of Hospital Infection 47 (Supplement): S I-S82.

Wilson, J. ( 1995 ) Infection Control in Clinical Practice. London: Bailliere Tindall. 